Vol. 45 (1992) [237-240]

\title{
A NOTE ON BOUNDS ON THE MINIMUM AREA OF CONVEX LATTICE POLYGONS
}

\section{Charles J. Colbourn and R.J. Simpson}

The minimum area $a(v)$ of a $v$-sided convex lattice polygon is known to satisfy $\left(\begin{array}{l}v \\ 2\end{array}\right) \leqslant a(2 v) \leqslant\left(\begin{array}{l}v \\ 3\end{array}\right)-v+1$. We conjecture that $a(v)=c v^{3}+o\left(v^{3}\right)$, for $c$ a constant; we prove that $a(v) \leqslant(15 / 784) v^{3}+o\left(v^{3}\right)$, and that for some positive constant $c$, $a(v) \geqslant c v^{2.5}$.

\section{Convex lattice Polygons}

A convex lattice polygon is a polygon whose vertices are points on the integer lattice with interior angles all convex. A lattice polygon with $v$ vertices is a $v$-gon. The minimum area of a convex lattice $v$-gon is denoted $a(v)$. The function $a(v)$ has been studied by Arkinstall [1], Rabinowitz [2] and Simpson [3]. Values of $a(v)$ are known exactly for $v \leqslant 10$ and $v \in\{12,13,14,16,18,20,22\}$. For general $v$, only bounds are known. Rabinowitz [2] established that $a(2 n) \leqslant\left(\begin{array}{c}n \\ 3\end{array}\right)-n+1$. Simpson [3] proved that $a(2 n) \geqslant\left(\begin{array}{l}n \\ 2\end{array}\right)$, and that

$$
\lfloor(a(2 n+2)+a(2 n)) / 2\rfloor+1 / 2 \leqslant a(2 n+1) \leqslant a(2 n+2)-1 / 2 .
$$

Together these imply that for all $v, a(v)>(1 / 8) v^{2}+o\left(v^{2}\right)$, and $a(v)<(1 / 48) v^{3}+$ $o\left(v^{3}\right)$.

In this note, we improve both the upper and the lower bound on $a(v)$, to prove that:

THEOREM 1.1. The minimum area of a convex lattice $v$-gon, $a(v)$, satisfies:

$$
c v^{2.5}<a(v)<(15 / 784) v^{3}+o\left(v^{3}\right)
$$

for $c$ a positive constant.

In section 2, we prove the upper bound and in section 3, we prove the lower bound. We conjecture that $a(v)=c^{\prime} v^{3}+o\left(v^{3}\right)$ for some positive constant $c^{\prime}$, hence motivating interest in specific constants $c^{\prime}$ for which $a(v)<c^{\prime} v^{3}+o\left(v^{3}\right)$.

\section{Received 18th March 1991}

This work was carried out whilst the first author was on leave from University of Waterloo, Canada. Research of the first author is supported in part by NSERC Canada under grant number A0579, and this research was supported in part by a grant from the Australian Research Council. Thanks to Stan Devitt for helpful discussions.

Copyright Clearance Centre, Inc. Serial-fee code: 0004-9729/92 \$A2.00+0.00. 
The relation between the case with odd $v$ and the case with even $v$ ensures that we need only treat the cases with $v$ even; henceforth, we write $v=2 n$. Simpson [3] proved that $a(2 n)$ is the same as the solution to an easily stated optimisation problem; we recall his formulation next. An admissible $n$-sequence is a sequence of $\boldsymbol{n}$ vectors with non-negative integer entries, $\left[v_{i}=\left(x_{i}, y_{i}\right), 1 \leqslant i \leqslant n\right]$, satisfying $y_{i} x_{j}-x_{i} y_{j}>0$ for $1 \leqslant i<j \leqslant n$. Simpson shows that without loss of generality, we can take $\mathbf{v}_{1}=(0,1)$ and $\mathbf{v}_{n}=(1,1)$.

ThEOREM 1.2. [3] For any admissible $n$-sequence $\left[\mathbf{v}_{i}=\left(x_{i}, y_{i}\right), 1 \leqslant i \leqslant n\right]$,

$$
a(2 n) \leqslant \sum_{i=1}^{n} \sum_{j=i+1}^{n}\left(y_{i} x_{j}-x_{i} y_{j}\right)
$$

moreover, $a(2 n)$ equals the minimum of the right hand side over all admissible $n$ sequences.

We employ this alternative characterisation of $a(2 n)$ in determining new upper and lower bounds.

\section{THE UPPER BOUND}

The upper bound relies on the explicit construction of an infinite family of admissible $n$-sequences.

LEMMA 2.1. $a(2 n) \leqslant 15 / 98 n^{3}+o\left(n^{3}\right)$.

PROOF: Since $a(2 n)<a(2 n+2)$, it suffices to construct admissible $n$-sequences realising the bound when $n \equiv 4(\bmod 14)$. Write $n=14 t+4$, and form an admissible $n$ sequence containing the vectors $(0,1),(1,1),\{(1, x): 2 \leqslant x \leqslant 10 t+2\}$ and $\{(2,2 x+1)$ : $3 t+1 \leqslant x \leqslant 7 t+1\}$. Applying Theorem 1.2 then establishes that

$$
\begin{aligned}
a(2 n) \leqslant 1 & +\sum_{i=2}^{10 t+2} i+\sum_{i=3 t+1}^{7 t+1}(2 i+1)+\sum_{i=1}^{10 t} \sum_{j=1}^{i} j+ \\
& \sum_{i=1}^{4 t} \sum_{j=1}^{i} 4 j+\sum_{\ell=-2 t}^{2 t}\left[1+\sum_{j=1}^{5 t-\ell}(2 j-1)+\sum_{j=1}^{5 t+\ell}(2 j+1)\right]
\end{aligned}
$$

which yields $a(2(14 t+4)) \leqslant 420 t^{3}+270 t^{2}+71 t+7$ for $t \geqslant 1$. The right hand side of the inequality determines the contribution to equation (1) of the pairs involving $(0,1)$ or $(1,1)$, then the pairs of vectors both of whose first components are 1 , then those whose first components are both 2, and finally those having one first component 1 and the other 2. Substituting $t=(n-4) / 14$ in the above gives the required bound. 


\section{THE LOWER BOUND}

The lower bound is obtained by establishing, for every convex lattice $2 n$-gon, a lower bound on the double sum in Theorem 1.2.

LEMMA 3.1. There is a positive constant $c$ for which $a(2 n) \geqslant c n^{2.5}$.

Proof: Suppose that $\left[v_{1}, \ldots v_{n}\right]$ is an admissible $n$-sequence with $v_{0}=(0,1)$ and $\mathbf{v}_{n}=(1,1)$. Consider the contribution to equation (1) arising from pairs containing $(0,1)$ or $(1,1)$. This contribution is

$$
1+\sum_{i=2}^{n-1}\left(x_{i}+y_{i}-x_{i}\right)
$$

which is one less than the sum $S$ of the $y$-components of the vectors of the sequence. Now to bound $S$, let $\ell_{i}$ be the number of vectors whose $y$ component is $i$; since the sequence is admissible, and $(1,1)$ is the last vector of the sequence, we have

$$
\ell_{i}<i \quad \text { for } i>1 \text {. }
$$

Clearly we have

$$
\sum_{i=1}^{\infty} \ell_{i}=n-1
$$

and

$$
\sum_{i=1}^{\infty} i \cdot \ell_{i}=S
$$

It is not hard to see that the left hand side of (4) is minimised subject to (2) and (3) when $\ell_{1}=1, \ell_{i}=i-1$ for $2 \leqslant i \leqslant k-1$ and $\ell_{k}=n-1-\sum_{i=1}^{k-1} \ell_{i}$, for a positive integer $k$ such that $0 \leqslant \ell_{k}<k$. Then (3) gives

$$
n-1=\left(k^{2}-3 k+2\right) / 2+1+\ell_{k},
$$

which implies that

$$
k \gg n^{0.5}
$$

From (4) we then have

$$
\begin{aligned}
S & \geqslant 1+\sum_{i=2}^{k-1} i(i-1)+k \ell_{k} \\
& =1+\left(\begin{array}{l}
k \\
3
\end{array}\right)+k \ell_{k} \\
& >10 c_{1}(n-1)^{1.5}
\end{aligned}
$$


for some constant $c_{1}$. We now show inductively that $a(2 n)>c_{1} n^{2.5}+O(n)$. Suppose that this holds for $n-1$. Considering in equation (1) the contribution $S_{0}$ of $(0,1)$ with a second vector, and the contribution $S_{1}$ of $(1,1)$ with a second vector, we have $S_{0}+S_{1}-1=S$, and hence one of these partial sums is at least $5 c_{1}(n-1)^{1.5}$. Using the induction hypothesis and the binomial expansion of $(1+x)^{2.5}$, we have

$$
\begin{aligned}
a(2 n) & \geqslant a(2(n-1))+5 c_{1}(n-1)^{1.5} \\
& \geqslant c_{1}\left[(n-1)^{2.5}+5(n-1)^{1.5}\right]+O(n) \\
& =c_{1}((n-1)+1)^{2.5}+O(n) .
\end{aligned}
$$

Hence $a(2 n) \geqslant c n^{2.5}$ for some positive constant $c$.

The lower bound would be improved by establishing that there is a fixed constant $\alpha>.5$ such that every admissible $n$-sequence has some vector $\mathrm{x}$ for which contribution to equation (1) of vector pairs $\{\{x, y\}: y \neq x\}$ is at least $c n^{1+\alpha}$. (Lemma 3.1 essentially shows that this statement holds with $\alpha=.5$.) This would give a lower bound that is $c^{\prime} n^{2+\alpha}$ on $a(2 n)$.

\section{REFERENCES}

[1] J.R. Arkinstall, 'Minimal requirements for Minkowski's theorem in the plane I', Bull. Austral. Math. Soc. 22 (1980), 259-274.

[2] S. Rabinowitz, 'On the number of lattice points inside a convex lattice $n$-gon', Congr. Numer. 73 (1990), 99-124.

[3] R.J. Simpson, 'Convex lattice polygons of minimum area', Bull. Austral. Math. Soc. 42 (1990), 353-367.

School of Mathematics and Statistics

Curtin University of Technology

GPO Box U 1987

Perth WA 6001

Australia 